\title{
Combining Radiomics and CNNs to Classify Benign and Malignant GIST
}

\author{
Tianqi Zhuo ${ }^{1,2}$, Xin $\mathrm{Li}^{1,2}$ and Hong Zhou ${ }^{1,2, \text { a) }}$ \\ ${ }^{1}$ College of Biomedical Engineering \& Instrument Science, Zhejiang University, China. \\ ${ }^{2}$ Key Laboratory for Biomedical Engineering of Ministry of Education, Zhejiang University of China \\ a) Corresponding Author: zhouh@mail.bme.zju.edu.cn
}

\begin{abstract}
This paper studies the classification of gastrointestinal stromal tumor (GIST) with machine learning and radiomics methods. The highlight of the paper is that combining features extracted from computed tomography (CT) images by radiomics and convolutional neural networks (CNNs) are used in classification task. It's different from the previous radiomics-only methodology including tumor intensity, tumor shape, tumor texture and wavelet features. In the paper, deep neural networks (DNN) U-Net and Res-Net realize high-level features extraction. The classifiers based on random forest (RF) and gradient boosting decision tree (GBDT) are trained with combining features extracted from computed tomography images. The experiments show that the combining features are effective biomarkers to discriminate benign and malignant GIST. The performance (accuracy $81 \%$, precision $78 \%$, recall $83 \%$ ) of the classifier is validated on 50 instances.
\end{abstract}

Key words: GIST, combining features, radiomics, DNN, classification, RF, GBDT.

\section{INTRODUCTION}

GIST is originated in Cajal stromal cells and express tyrosine kinase growth factor receptor CD117 and CD34 1. The incidence of GIST is about 10 to 20 cases per million people, of which $20 \%$ to $30 \%$ are malignant 2 . The early detection rate of GIST is unsatisfactory due to the concealed appearance and the lack of specific oncological indicators. Due to the obvious heterogeneity and diverse biological behaviors, it's a vital clinical problem 3 .

As tumors are spatially and temporally heterogeneous, radiomics was proposed which indicated medical images contained a lot of effective information 4. With radiomics, medical images can be converted to data including intensity, shape, texture and wavelet features. These medical image features provide a novel powerful tool for pre-diagnosis and prognosis of radiation therapy. Cui et al. 5 constructed a prognosis model with radiomics features for advanced pancreatic cancer patients' radiation therapy. It's significantly better than other models without radiomics features. Timothy et al. 6 proposed a prediction model for local control effect of tumor, Sarah et al. 7 built a recurrence prediction model for lung cancer patients. Radiomics demonstrated its strong ability to make the clinical decisions for tumor radiotherapy.

Radiogenomics was proposed by Baumann et al. 8. The sensitivity of tumor tissue to radiotherapy was researched, however, the connection of image characteristics and gene expression was not noticed. Radiogenomics is the bridge between macroscopic images and microscopic genetic information. Then, Segal 9, Kuo 10 and Rutman 11 published their own results about the relationship of medical image features and gene expression. Segal et a19. studied the relevance of the radiomics features and the gene expression profile. With radiomics, some recent researches demonstrate that the gene KDM5C and SETD2 are relevant to the size of tumor, the gene mTOR is related to the location of tumor 12. Huang et al. 13 utilized a radiomics model to estimate the probability of Colorectal cancer lymph node metastasis. Radiogenomics can guide the treatment, especially about the sensitivity and the tolerability of antitumor drugs. 
Recent years, the deep learning shows its capabilities in the computer vision $(\mathrm{CV})$ and natural language processing (NLP) 141516,17,18,19. It's a representation learning based on data 14. Compared with the handcrafted features, more high-level and abstract features are extracted automatically by deep learning methods. In the computer vision, the convolutional neural networks (CNNs) are widely used, such as AlexNet, VGGNet, GoogLeNet, ResNet, DenseNet and so on $14,15,16,17,18,19$. CheXNet was proposed by Rajpurkar et al. to detect pneumonia from chest X-rays 21 . And they extend the model to detect 14 diseases in the dataset ChestX-ray14 and achieve a state of the art result. UNet, 3D U-Net and V-Net are used in segmentation to locate region of interest (ROI) or volume of interest (VOI) $22,23,24$.

In this paper, we study the classification of GIST with machine learning and radiomics methods. We innovatively introduce the abstract features extracted by $\mathrm{CNNs}$ and combine the radiomics features to achieve differentiation between benign and malignant GIST. The classification task is realized by random forest and gradient boosting decision tree. The performance of the classifier is satisfying and robust. The accuracy, precision and recall are $81 \%$, $78 \%$ and $83 \%$, respectively.

\section{DATASET AND METHODS}

\section{Dataset}

Ethical approval was obtained for this retrospective analysis. The GIST patients' CT images were provided by Fudan University Cancer Hospital. In total, 101 patients compose the primary cohort. The ROI of every CT image was located by two professional radiologists with ITK-SNAP tool 25. And the ROIs of one GIST patient compose the VOI for radiomics. The ROI and the VOI of an example are shown in Fig 1 and Fig 2. The original level of GIST was labeled by NIH2008, WHO and AFIP, respectively. In this research, the GISTs identified 'low' level, '2' or less level and ' $10 \%$ ' or less by NIH2008, WHO and AFIP, respectively, are labeled with ' 0 ' and the rest are labeled with ' 1 '. A fraction of GISTs annotated with binary label are shown in Table 1.

TABLE 1. A fraction of GISTs are annotated with the original label and the binary label.

\begin{tabular}{ccccc}
\hline ID & NIH2008 & WHO & AFIP & Binary Label \\
\hline 10665237 & middle & $3 \mathrm{a}$ & $3.60 \%$ & 1 \\
10707917 & high & $6 \mathrm{a}$ & $55 \%$ & 1 \\
10724764 & low & 2 & $1.90 \%$ & 0 \\
10747679 & low & 2 & $4.30 \%$ & 0 \\
10760831 & high & $6 \mathrm{a}$ & $55 \%$ & 1 \\
10770397 & low & 2 & $1.90 \%$ & 0 \\
\hline
\end{tabular}

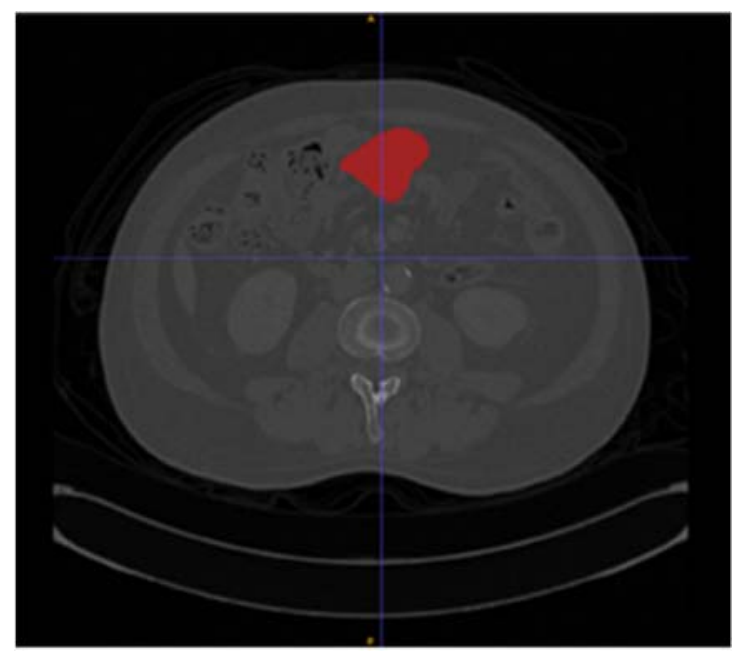

FIG 1. The ROI of one CT image. 


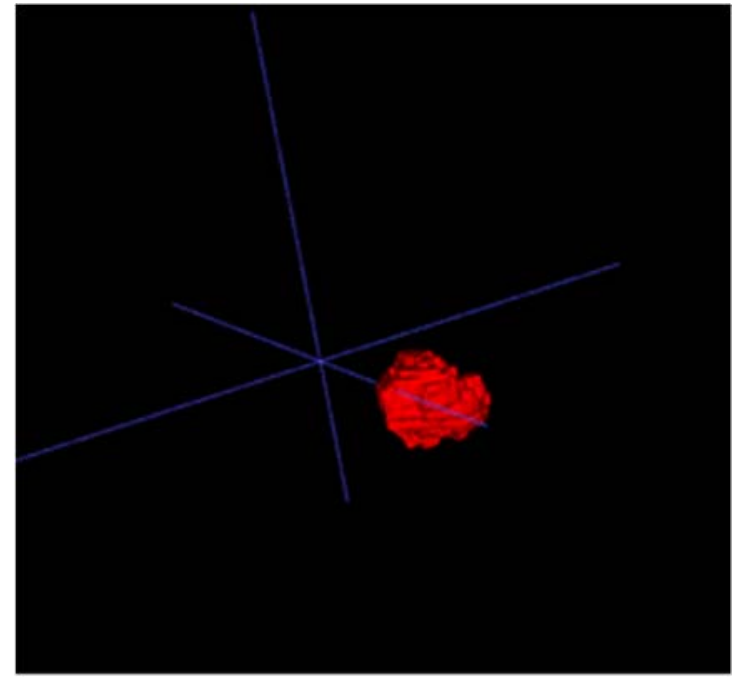

FIG 2. The VOI of one GIST patient. The VOI composes of ROI in one GIST patient' CT images.

\section{Extract Radiomics Features and DNN Features}

The radiomics features are extracted by an opensource software pyradiomics automatically. In this paper, the radiomics features compose of First Order Features, Shape Features, Gary Level Co-occurrence Matrix Features, Gray Level Size Zone Matrix Features, Gray Level Run Length Matrix Features, Neighboring Gray Tone Difference Matrix and Gray Level Dependence Matrix.

The DNN features are extracted by ResNet-50 which has been pre-trained on a large-scale image dataset ImageNet. Some abstract features extracted by CNN is shown in Fig 3. The ultima DNN features compose of a 100-dimension vector. This process is shown in Fig 4.

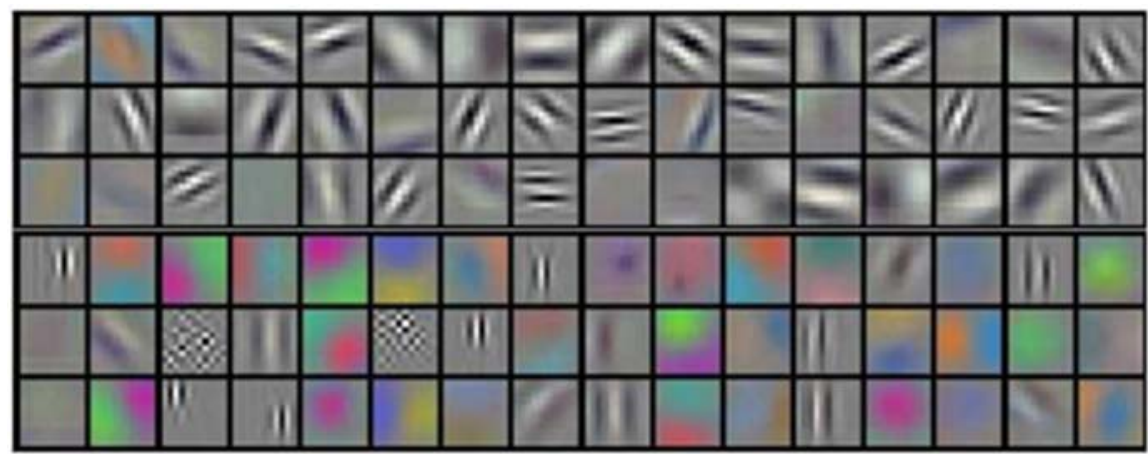

FIG 3. Some abstract features extracted by $\mathrm{CNN}$.

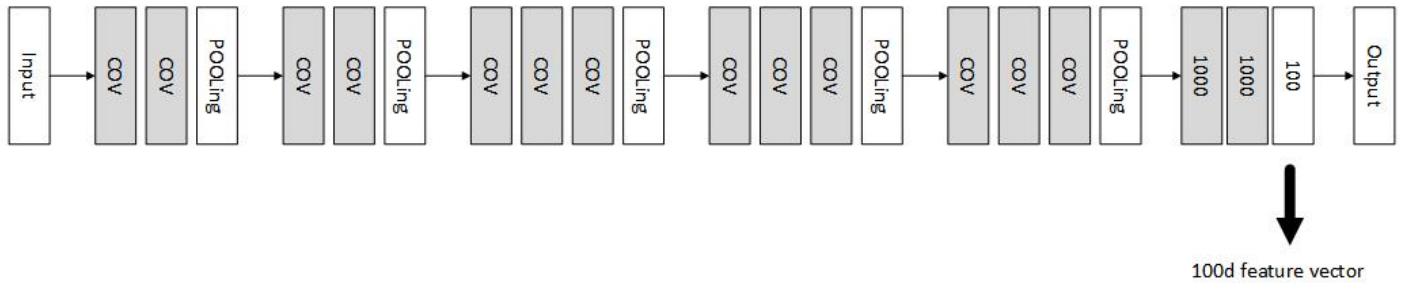

FIG 4. The extraction process of the 100-dimension DNN feature vector. 


\section{Select Features and Reduce Dimension}

Pearson's correlation coefficient is used to analyze the features correlation. The Pearson's correlation coefficient of two variable is defined as equation 1.

$$
\rho_{X, Y}=\frac{\operatorname{cov}(X, Y)}{\sigma_{X} \sigma_{Y}}=\frac{E\left[\left(X-\mu_{X}\right)\left(Y-u_{Y}\right)\right]}{\sigma_{X} \sigma_{Y}}
$$

$\rho$ is the correlation coefficient, cov is the covariation function, $\mathrm{E}$ is the expectation function, $\sigma$ is the standard deviation, $\mu$ is the average value. Usually, the equation 2 is used to estimate the Pearson's correlation coefficient.

$$
\mathrm{r}=\frac{\sum_{i=1}^{n}\left(X_{i}-\bar{X}\right)\left(Y_{i}-\bar{Y}\right)}{\sqrt{\sum_{i=1}^{n}\left(X_{i}-\bar{X}\right)^{2}} \sqrt{\sum_{i=1}^{n}\left(Y_{i}-\bar{Y}\right)^{2}}}
$$

$\mathrm{r}$ is the correlation coefficient, $\bar{X}, \bar{Y}$ is the average value.

\section{GIST Classification}

After dimension reduction, the remaining features compose the signature which is regarded as the input of the classifiers. The classifiers are based on linear regression (LR), RF, GBDT, DNN and multi-model, respectively. The performance of the classifiers is evaluated by receiver operating characteristic curve, confusion matrix, accuracy, precision and recall.

\section{RESULTS}

\section{Dimension Reduction Based on Features Correlation}

The Pearson's correlation coefficient matrix is shown in Fig 5. To insure the independency of features, 70\% features are chosen by Pearson's correlation coefficient. In addition, the features are binarized and rarefied which is shown in Table 2 .

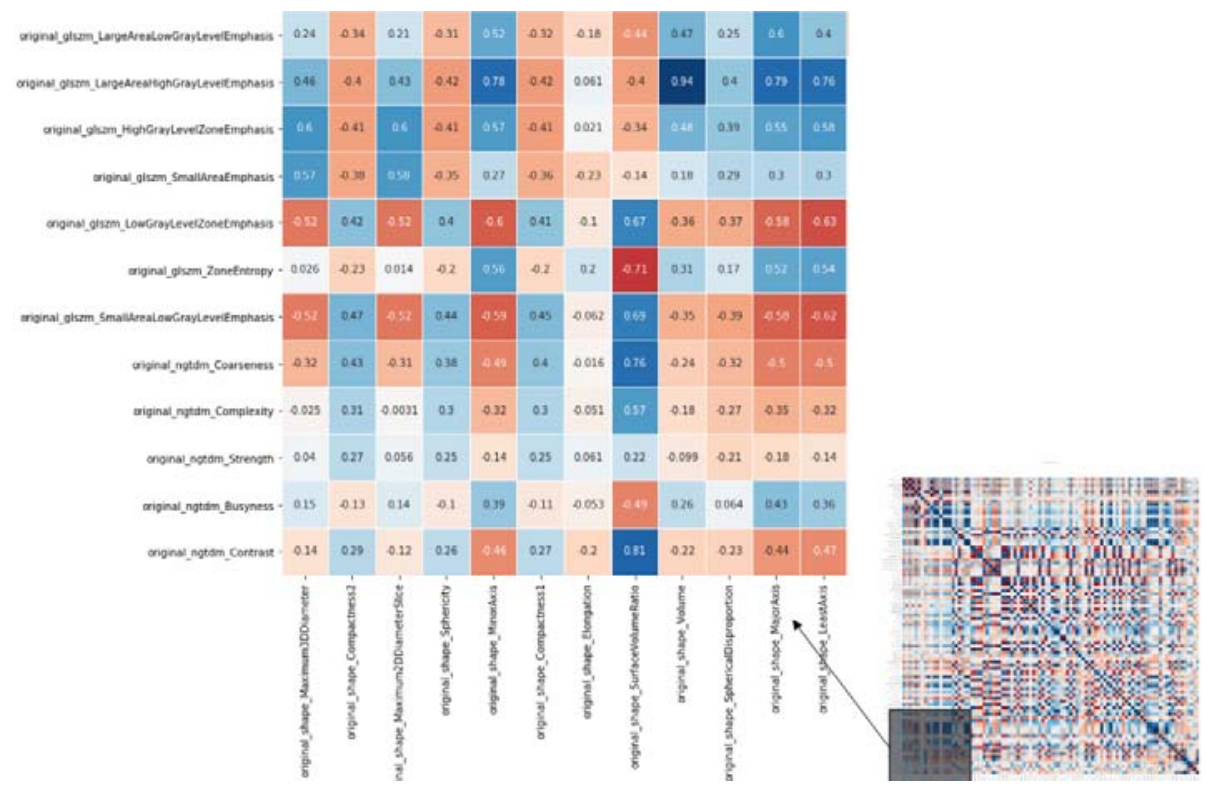

FIG 5. A fraction of Pearson's correlation coefficient matrix. 
TABLE 2. A fraction of sparse features.

\begin{tabular}{ccc}
\hline C_O_ngtdm_Complexity & C_O_ngtdm_Strength & C_O_ngtdm_Busyness \\
\hline 2 & 1 & 2 \\
0 & 0 & 3 \\
0 & 0 & 3 \\
3 & 2 & 0 \\
2 & 1 & 1 \\
\hline
\end{tabular}

\section{The Performance of Classifiers}

Classifiers include LR, GBTD, RF, DNN and multi-model. The details of the classifiers' performance are shown in the Table 3. The Fig 6 is shown the performance of the multi-model on the test data set by confusion matrix. The ROC curve of the multi-model on the test data set is shown in FIG 7.

TABLE 3. The performance of the classifiers.

\begin{tabular}{cccc}
\hline Model & Accuracy & Recall & Precision \\
\hline Radiomics+LR & $52 \%$ & - & - \\
Radiomics+GBDT & $61 \%$ & - & - \\
Radiomics+RF & $59 \%$ & - & - \\
DNN & $60 \%$ & - & - \\
DNN+Radiomics+GBDT & $67 \%$ & - & - \\
DNN+Radiomics_multi-model & $81 \%$ & $83 \%$ & $78 \%$ \\
\hline
\end{tabular}

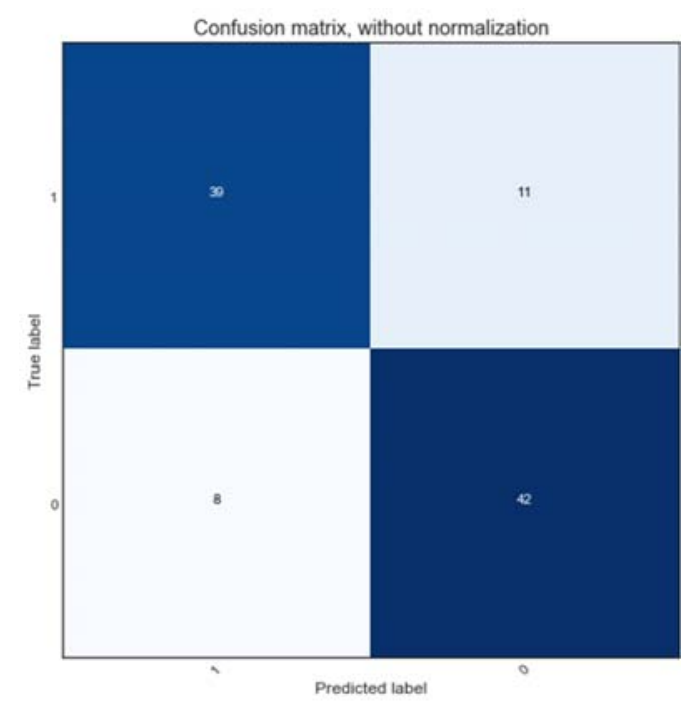

FIG 6. The confusion matrix of the multi-model on the test data set. 


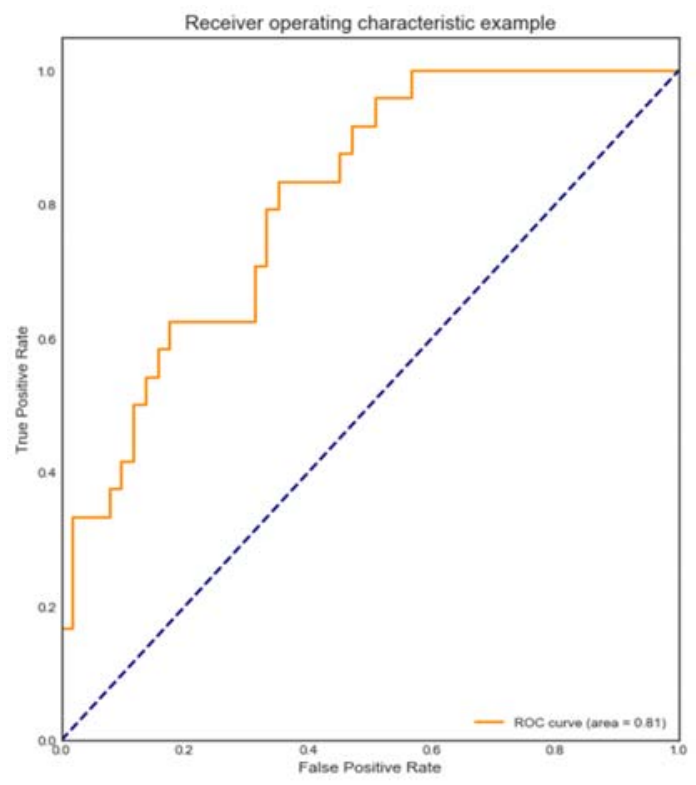

FIG 7. The ROC curve of the multi-model on the test data set. Its area under curve is 0.81 .

\section{DISCUSSION}

GIST is a significant threat. The early detection rate of GIST is unsatisfactory because of the concealed appearance and the lack of specific oncological indicators. Due to the obvious heterogeneity and diverse biological behaviors, it's a vital clinical problem. In this paper, the radiomics and deep learning are utilized to distinguish the benign and malignant GIST. The features of CT images are extracted by radiomics and DNN. After features selection, a feature signature is defined as the input for the classifiers. The U-Net and ResNet are utilized to extract abstract and highlevel features from CT images. The multi-classifier based on random forest and gradient boosting decision tree has a satisfying performance on test data set.

In this paper, because the small data set is a great difficulty, the transfer learning is used to extracted high-level features. The preceding U-Net and ResNet have been pre-trained on ImageNet data set. In future studies, more GIST data will be collected to achieve a better generalization performance on unseen GIST CT images.

\section{REFERENCES}

1. Miettinen M, Majidi M, Lasota J. Pathology and diagnostic criteria of gastrointestinal stromal tumors (GISTs): a review. Eur J Cancer. 2002;38(suppl 5): S39-S51.

2. Miettinen M, Lasota J. Gastrointestinal stromal tumors - definition, clinical, histological, immunohistochemical, and molecular genetic features and differential diagnosis. Virchows Arch. 2001; 438:1-12.

3. Joensuu H, DeMatteo RP. The management of gastrointestinal stromal tumors: a model for targeted and multidisciplinary therapy of malignancy. Annu Rev Med. 2012; 63:247-258.

4. Lambin P, Rios-Velazquez E, Leijenaar R, et al. Radiomics: extracting more information from medical images using advanced feature analysis. Eur J Cancer. 2012. 48(4): 441-6.

5. Cui Y, Song J, Pollom E, et al. Radiomic Analysis of FDG-PET Identifies Novel Prognostic Imaging Biomarkers in Locally Advanced Pancreatic Cancer Patients Treated with SBRT[C]. ASTRO, 2015.

6. T.P.C. Yeung, G. Rodrigues, F. Lagerwaard, et al. Prediction of Stereotactic Radiosurgery Brain Metastasis Lesion Control Using Radiomic Features[C]. ASTRO, 2015.

7. Mattonen S.A, Tetar S, Palma D.A, et al. Imaging texture analysis for automated prediction of lung cancer recurrence after stereotactic radiotherapy[J]. Med Imaging, 2015, 2(4): 041010.

8. Baumann M, Holscher T, Begg AC. Towards genetic prediction of radiation responses: ESTRO's GENEPI project. Radiother Oncol. 2003. 69(2): 121-5. 
9. Segal E, Sirlin CB, Ooi C, et al. Decoding global gene expression programs in liver cancer by noninvasive imaging. Nat Biotechnol. 2007. 25(6): 675-80.

10. Kuo MD, Gollub J, Sirlin CB, Ooi C, Chen X. Radiogenomic analysis to identify imaging phenotypes associated with drug response gene expression programs in hepatocellular carcinoma. J Vasc Interv Radiol. 2007. 18(7): 821-31.

11. Rutman AM, Kuo MD. Radiogenomics: creating a link between molecular diagnostics and diagnostic imaging. Eur J Radiol. 2009. 70(2): 232-41.

12. Karlo CA, Di PPL, Chaim J, et al. Radiogenomics of clear cell renal cell carcinoma: associations between CT imaging features and mutations. Radiology. 2014. 270(2): 464-71.

13. Huang YQ, Liang $\mathrm{CH}$, He L, et al. Development and validation of a radiomics nomogram for preoperative prediction of lymph node metastasis in colorectal cancer. J Clin Oncol, 2016.

14. Krizhevsky A, Sutskever I, Hinton G E. ImageNet Classification with Deep Convolutional Neural Networks [J]. Advances in Neural Information Processing Systems, 2012, 25(2):2012.

15. Simonyan K, Zisserman A. Very deep convolutional networks for large-scale image recognition[J]. arXiv preprint arXiv:1409.1556, 2014.

16. Szegedy C, Liu W, Jia Y, et al. Going deeper with convolutions[C]/Proceedings of the IEEE conference on computer vision and pattern recognition. 2015: 1-9.

17. He K, Zhang $\mathrm{X}$, Ren $\mathrm{S}$, et al. Deep residual learning for image recognition[C]/Proceedings of the IEEE conference on computer vision and pattern recognition. 2016: 770-778.

18. Huang G, Liu Z, Weinberger K Q, et al. Densely connected convolutional networks[J]. arXiv preprint arXiv:1608.06993, 2016.

19. Russakovsky O, Deng J, Su H, et al. Imagenet large scale visual recognition challenge[J]. International Journal of Computer Vision, 2015, 115(3): 211-252.

20. Song, H.A.; Lee, S. Y. Hierarchical Representation Using NMF. Neural Information Processing. Lectures Notes in Computer Sciences 8226.

21. Rajpurkar P, Irvin J, Zhu K, et al. CheXNet: Radiologist-Level Pneumonia Detection on Chest X-Rays with Deep Learning[J]. arXiv preprint arXiv:1711.05225, 2017.

22. Ronneberger O, Fischer P, Brox T. U-net: Convolutional networks for biomedical image segmentation[C]//International Conference on Medical image computing and computer-assisted intervention. Springer, Cham, 2015: 234-241.

23. Çiçek Ö, Abdulkadir A, Lienkamp S S, et al. 3D U-Net: learning dense volumetric segmentation from sparse annotation[C]//International Conference on Medical Image Computing and Computer-Assisted Intervention. Springer, Cham, 2016: 424-432.

24. Milletari F, Navab N, Ahmadi S A. V-net: Fully convolutional neural networks for volumetric medical image segmentation[C]//3D Vision (3DV), 2016 Fourth International Conference on. IEEE, 2016: 565-571.

25. Paul A. Yushkevich, Joseph Piven, Heather Cody Hazlett, Rachel Gimpel Smith, Sean Ho, James C. Gee, and Guido Gerig. User-guided 3D active contour segmentation of anatomical structures: Significantly improved efficiency and reliability. Neuroimage 2006 Jul 1;31(3):1116-28. 